\title{
In vitro $\alpha$-glucosidase inhibitory activity of compounds isolated from mangrove Lumnitzera littorea leaves
}

\author{
Nguyen Thi Le Thuy ${ }^{1}$, Pham Thi Thuy ${ }^{2}$, Poul Erik Hansen ${ }^{3}$, Nguyen Kim Phi Phung ${ }^{2}$,*
}

\begin{abstract}
Introduction: Lumnitzera littorea grown at CanGio Mangrove Forest has been investigated. The present study reports the isolation, characterization and evaluation of the $\alpha$-glucosidase inhibitory activity of isolated compounds from Lumnitzera littorea leaves. Methods: Their structures were elucidated by spectroscopic methods (including MS, 1D and 2D-NMR) and comparison with values from the literature. From the $n$-hexane extract, nine compounds including lupeol (1), betulin (2), betulinic acid (3), oleanolic acid (4), corosolic acid (5), $\beta$-sitosterol (6), $\beta$-sitosterol 3-O$\beta$-D-glucopyranoside (7), stigmast-5-ene-3 $\beta$-O-(6-O-hexadecanoyl- $\beta$-D-glucopyranoside) (8), and stigmast-4-ene-3-one (9) were isolated and identified. Results: The results of the $\alpha$-glucosidase inhibitory activity showed thatcorosolic acid (5) and oleanolic acid (4) were the most potent, with $I C_{50}$ values of $17.86 \pm 0.42$ and $18.82 \pm 0.59 \mu \mathrm{g} / \mathrm{mL}$, respectively. Five of the other seven compounds exhibited inhibitory activity with $\mathrm{IC}_{50}$ values below $100 \mu \mathrm{g} / \mathrm{mL}$, and higher than the positive control acarbose $(127.64 \pm 0.64 \mu \mathrm{g} / \mathrm{mL})$.

Key words: Lumnizera littorea, mangrove plant, $\alpha$-glucosidase inhibitory activity
\end{abstract}

${ }^{1}$ Department of BioTechnology,

HoChiMinh city Open University,

Vietnam

${ }^{2}$ Department of Chemistry, University of Science, VNU-HCM

${ }^{3}$ Department of Science and Enviroment, Roskilde University, Denmark

\section{Correspondence}

Nguyen Kim Phi Phung, Department of Chemistry, University of Science, VNU-HCM

Email: kimphiphung@yahoo.fr

\section{History}

- Received: 2018-11-12

- Accepted: 2018-12-26

- Published: 2019-01-07

\section{DOI :}

https://doi.org/10.32508/stdj.v22i1.1009

\section{Check for updates}

\section{Copyright}

(.) VNU-HCM Press. This is an openaccess article distributed under the terms of the Creative Commons Attribution 4.0 International license.

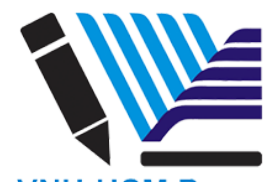

VNU-HCM Press

\section{INTRODUCTION}

Diabetes is a chronic disease associated with unusually high levels of glucose in the blood. The goal of diabete therapy is the maintenance of normal blood glucose levels after a meal. Postprandial hyperglycemia plays an important role in the development of type 2 diabetes and its complications. One of the therapeutic approaches for decreasing blood glucose rise after a meal is to slow down the absorption of glucose by inhibition of carbohydrate hydrolyzing enzymes, such as $\alpha$-glucosidase. $\alpha$-Glucosidase is an intestinal enzyme that breaks down $\alpha-1,4$ linked polysaccharides to $\alpha$-glucose, which leads to the high blood sugar levels. The development of an $\alpha$-glucosidase inhibitor derived from natural products is an important contribution for the treatment of diabetes.

Lumnitzera littorea, a woody tree of the Combretaceae family, grows at the Can Gio Mangrove Forest in Vietnam. The antimicrobial activities of $n$-hexane, ethyl acetate and methanol extracts of leaves of this species were evaluated against six human pathogenic microbes and the former extract was the most active $^{1}$. Our published research showed that the $\alpha$ glucosidase inhibitory activity on all extracts and isolated flavonoids from the leaves of Lumnitzera littorea were very strong ${ }^{2}$. The aim of this study was to isolate phytoconstituents and evaluate the inhibition of $\alpha$ glucosidase activity of the compounds isolated from the $n$-hexane extract of $L$. littorealeaves.

\section{METHODS}

\section{Plant materials}

Leaves of Lumnitzera littorea (Jack) Voigt (Combretaceae) Figure 1were collected at Can Gio Mangrove Forest of Ho Chi Minh city, Viet Nam in August of 2014. The scientific name of plant was authenticated by Dr. Pham Van Ngot, Faculty of Biology, Ho Chi Minh City University of Pedagogy. A voucher specimen (No US-B012) was deposited in the herbarium of the Department of Organic Chemistry, University of Science.

\section{GENERAL EXPERIMENTAL PROCEDURES}

The NMR spectra were recorded on a Bruker Avance III spectrometer at $500 \mathrm{MHz}$ for ${ }^{1} \mathrm{H}$ NMR and 125 $\mathrm{MHz}$ for ${ }^{13} \mathrm{C}$ NMR spectra. ESI-MS were performed on a Shimadzu +IDA TOF MS. TLC was performed on silica gel $60 \mathrm{~F}_{254}$ (Merck, Darmstadt, Germany). Gravity column chromatography was performed on silica gel $60(0.040-0.063 \mathrm{~mm}$, Merck, Darmstadt, Germany) and Sephadex LH-20 (GE Healthcare BioScience AB, Uppsala, Sweden). $\alpha$-Glucosidase (EC 3.2.1.20) from Saccharomyces cerevisiae (750 UN) and $p$-nitrophenyl- $\alpha$-D-glucopyranoside were purchased from Sigma Chemical Co. (St. Louis, MO, USA). Acarbose and dimethyl sulfoxide were obtained from 


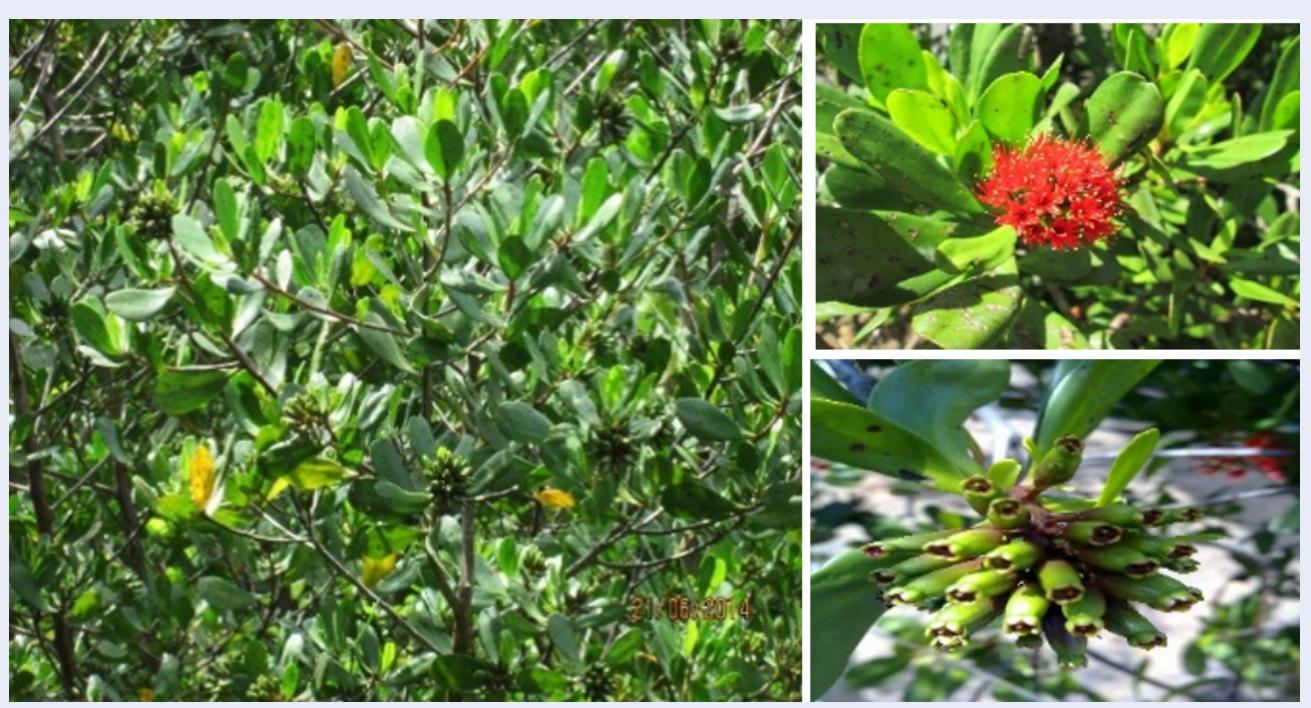

Figure 1: Lumnitzera littorea (Jack) Voigt.

Merck (Darmstadt, Germany). Other chemicals were of the highest grade available.

\section{EXTRACTION AND ISOLATION}

The fresh leaves were washed under running tap water to remove all sandy particles and epiphytes and then were dried and ground into fine powder. The powder $(15,000 \mathrm{~g})$ was exhaustively extracted with ethanol at room temperature by the method of maceration. After filtration, the ethanol solution was evaporated to dryness under reduced pressure to yield a crude ethanol residue $(1,000 \mathrm{~g})$. This crude was applied to a silica gel solid phase extraction, eluted consecutively with $n$-hexane, ethyl acetate, and finally with ethanol. After evaporation under reduced pressure, three extracts were obtained: $n$-hexane (100 g), ethyl acetate (250 g), and ethanol (550 g).

The $n$-hexane extract ( $100 \mathrm{~g}$ ) was fractionated by silica gel column chromatography using a mixture of $n$-hexane-ethyl acetate (98:2 to $0: 100)$ to yield five fractions $(\mathrm{H} 1-\mathrm{H} 5)$. Fraction $\mathrm{H} 2(52.5 \mathrm{~g})$ was applied to a silica gel column and eluted with chloroform:methanol (stepwise, 98:2 to 50:50) to give 6 subfractions (H21-H26). Subfraction H21 was rechromatographed on a silica gel column using chloroform:ethylacetate (stepwise 98:2 to 0:100), and then purified by Sephadex LH-20 chloroform:methanol (1:1) to obtain compound $\mathbf{1}(20 \mathrm{mg})$. Subfraction $\mathrm{H} 23$ was further chromatographed on Sephadex LH20 chloroform:methanol (1:1) to give two compound : compound 8 (10 mg) and $\mathbf{9}(15 \mathrm{mg})$.

Fraction H3 was further separated on a silica gel column and eluted with chloroform:methanol (stepwise,
9:1 to $0: 100)$ to yield four fractions $(\mathrm{H} 31-\mathrm{H} 34)$. Subfraction H31 was subjected to Sephadex LH-20 chloroform:methanol (1:1), then separated on a silica gel Rp18 with water:methanol:acetone $(2: 3: 5)$ to obtain three compounds, such as compound 2 (10 mg), 3 (15 $\mathrm{mg}$ ), and 4 (5 mg).

Fraction H4 was applied to a silica gel column and eluted with chloroform:methanol:water (14:6:1) to yield five fractions (H41-H45). Subfraction H41 was further separated on Sephadex LH-20 chloroform:methanol (1:1) to give compound $5(15 \mathrm{mg})$ and 6 (25 mg). Subfraction H43 was rechromatographed on a silica gel column with $n$-hexane:chloroform (stepwise, 95:5 to 50:50) to obtain compound 7 (20 $\mathrm{mg}$ ).

\section{In vitro $\alpha$-glucosidase inhibitory assay}

The $\alpha$-glucosidase inhibitory activity was evaluated on all compounds according to the method of Apostolidis et al. ${ }^{3}$. A reaction mixture containing $60 \mu \mathrm{L}$ of $100 \mathrm{mM}$ phosphate buffer ( $\mathrm{pH} 6.8$ ), $20 \mu \mathrm{L}$ of sample (at the different concentrations), and $100 \mu \mathrm{L}$ of 200 $\mu \mathrm{M} p$-nitrophenyl- $\alpha$-D-glucopyranoside solution (in $100 \mathrm{mM}$ phosphate buffer) was incubated in 96-well plates at $37^{\circ} \mathrm{C}$ for $10 \mathrm{~min}$. Then, $20 \mu \mathrm{L}$ of $0.3 \mathrm{U} / \mathrm{mL}$ $\alpha$-glucosidase in the phosphate buffer was added to the mixture. The reaction mixtures were incubated at $37^{\circ} \mathrm{C}$ for $10 \mathrm{~min}$. Then, the reaction was stopped by adding $20 \mu \mathrm{L}$ of $50 \mathrm{mM} \mathrm{NaOH}$. Absorbances were recorded at $405 \mathrm{~nm}$ by a microplate reader and compared to a control which had $20 \mu \mathrm{L}$ of buffer solution in place of the sample. Acarbose was used as a positive 
control. The $\alpha$-glucosidase inhibitory activity was expressed as $\%$ inhibition and was calculated as follows: $\%$ Inhibition $=\left[\left(\mathrm{A}_{\text {control }}-\mathrm{A}_{\text {sample }}\right) / \mathrm{A}_{\text {control }}\right]^{\star} 100$ The inhibitory concentration $\left(\mathrm{IC}_{50}\right)$ for each sample was calculated using a regression analysis from the graph plotting scavenging activity against concentration. All experiments were carried out in triplicate and the results were expressed as the mean $\pm \mathrm{SD}$ of three determinations.

\section{Statistical analysis}

All assays were conducted in triplicate. Statistical analyses were performed with Statgraphics Plus Professional 16.0.03 for an analysis of variance (ANOVA), followed by Duncan's test. Differences at $\mathrm{P}<0.05$ were considered significant.

\section{RESULTS}

\section{Structural elucidation}

The phytochemical study of Lumnitzera littorea led to the isolation and identification of nine compounds whose structures are shown in Figure 2. The spectral properties of these known compounds, including ${ }^{1} \mathrm{H}-\mathrm{NMR}$ and ${ }^{13} \mathrm{C}-\mathrm{NMR}$ data, were identical to those previously described in the literature.

Lupeol (1): white powder, ESI-MS $m / z: 426.0[\mathrm{M}]^{+}$ for $\mathrm{C}_{30} \mathrm{H}_{50} \mathrm{O} .{ }^{1} \mathbf{H}-\mathrm{NMR}$ ( $500 \mathrm{MHz}, \mathrm{CDCl}_{3}$ ) d (ppm): 4.68 (brs, H-29a), 4.56 (brs, H-29b), 3.18 (dd, 11.5, $5.0 \mathrm{~Hz}, \mathrm{H}-3), 1.68$ ( $s, \mathrm{H}-30), 1.03$ (s, H-26), 0.96 (s, $\mathrm{H}-23), 0.94$ ( $s, \mathrm{H}-27), 0.83$ (s, H-25), 0.79 ( $s, \mathrm{H}-28)$ and $0.76(s, \mathrm{H}-24),{ }^{13} \mathrm{C}-\mathrm{NMR}\left(125 \mathrm{MHz}, \mathrm{CDCl}_{3}\right) \mathrm{d}$ (ppm): 151.1 (C-20), 109.5 (C-29), 79.1 (C-3), 55.5 (C-5), 50.6 (C-9), 48.5 (C-18), 48.1 (C-19), 43.1 (C14), 43.0 (C-17), 41.0 (C-8), 40.2 (C-22), 39.0 (C-4), 38.9 (C-1), 38.2 (C-13), 37.3 (C-10), 35.7 (C-16), 34.4 (C-7), 30.0 (C-21), 28.1 (C-23), 27.6 (C-2, C-15), 25.3 (C-12), 21.1 (C-11), 19.5 (C-30), 18.5 (C-6), 18.2 (C28), 16.3 (C-25), 16.1 (C-26), 15.5 (C-24) and 14.7 (C27).

Betulin (2): white powder, ESI-MS $m / z$ : 443.44 $[\mathrm{M}+\mathrm{H}]^{+}$for $\mathrm{C}_{30} \mathrm{H}_{50} \mathrm{O}_{2}$. ${ }^{1} \mathbf{H}-\mathrm{NMR}(500 \mathrm{MHz}$, DMSO- $\left.d_{6}\right) \mathrm{d}$ (ppm): $4.68(d, 2.0 \mathrm{~Hz}, \mathrm{H}-29 \mathrm{a}), 4.58$ (brs, H-29b), 3.80 (d, $10.5 \mathrm{~Hz}, \mathrm{H}-28 \mathrm{a}), 3.33$ (d, 11.0 $\mathrm{Hz}, \mathrm{H}-28 \mathrm{~b}), 3.18$ (dd, 11.0, $4.0 \mathrm{~Hz}, \mathrm{H}-3), 2.38$ ( $m, \mathrm{H}-$ 18), 1.68 (brs, H-30), 1.02 ( $s, \mathrm{H}-25), 0.98$ ( $s, \mathrm{H}-27)$, $0.97(s, \mathrm{H}-23), 0.82(s, \mathrm{H}-26)$ and $0.76(s, \mathrm{H}-24),{ }^{13} \mathrm{C}-$ NMR (125 MHz, DMSO- $d_{6}$ ) d (ppm): 150.6 (C-20), 109.8 (C-29), 79.1 (C-3), 60.7 (C-28), 55.5 (C-5), 50.6 (C-9), 48.9 (C-18), 47.9 (C-19, C-17), 42.9 (C-14), 41.1 (C-8), 39.0 (C-1), 38.9 (C-4), 37.5 (C-10), 37.3 (C-13), 34.4 (C-7), 34.1 (C-22), 29.9 (C-21), 29.3 (C16), 28.1 (C-23), 27.6 (C-2), 27.2 (C-15), 25.4 (C-12),
21.0 (C-11), 19.2 (C-30), 18.5 (C-6), 16.3 (C-25), 16.1 (C-26), 15.5 (C-24) and 14.9 (C-27).

Betulinic acid (3): white powder, ESI-MS $m / z: 455.38$ $[\mathrm{M}-\mathrm{H}]^{-}$corresponding for $\mathrm{C}_{30} \mathrm{H}_{48} \mathrm{O}_{3} .{ }^{\mathbf{1}} \mathbf{H}-\mathbf{N M R}$ (500 MHz, $\mathrm{CDCl}_{3}$ ) d (ppm): 4.74 (brs, H-29a), 4.61 (brs, H-29b), 3.19 (dd, 11.0, $4.5 \mathrm{~Hz}, \mathrm{H}-3), 3.00$ ( $m, \mathrm{H}-$ 19), 1.69 ( $s, \mathrm{H}-30), 0.98$ ( $s, \mathrm{H}-26), 0.96$ ( $s, \mathrm{H}-27), 0.93$ $(s, \mathrm{H}-23), 0.82(s, \mathrm{H}-25)$ and $0.75(s, \mathrm{H}-24),{ }^{13} \mathrm{C}-\mathrm{NMR}$ $\left(125 \mathrm{MHz}, \mathrm{CDCl}_{3}\right) \mathrm{d}$ (ppm): 180.5 (C-28), 150.6 (C20), 109.8 (C-29), 79.2 (C-3), 56.5 (C-17), 55.5 (C-5), 50.7 (C-9), 49.4 (C-19), 47.1 (C-18), 42.6 (C-14), 40.9 (C-8), 39.0 (C-4), 38.9 (C-1), 38.6 (C-13), 37.4 (C-10), 37.2 (C-22), 34.5 (C-7), 32.3 (C-16), 30.7 (C-15), 29.9 (C-21), 28.1 (C-23), 27.6 (C-2), 25.7 (C-12), 21.0 (C11), 19.5 (C-30), 18.4 (C-6), 16.3 (C-26), 16.2 (C-25), 15.5 (C-24) and 14.9 (C-27).

Oleanolic acid (4): white powder, $\mathrm{C}_{30} \mathrm{H}_{48} \mathrm{O}_{3}$. ${ }^{1} \mathbf{H}$ NMR (500 MHz, $\left.\mathrm{CDCl}_{3}\right) \mathrm{d}(\mathrm{ppm}): 5.29(t, 3.5 \mathrm{~Hz}, \mathrm{H}-$ 12), 3.22 ( $d d, 11.5,4.0 \mathrm{~Hz}, \mathrm{H}-3), 1.14(s, \mathrm{H}-27), 0.99$ ( $s$, $\mathrm{H}-29), 0.93$ (s, H-30), 0.91 ( $s, \mathrm{H}-23, \mathrm{H}-25), 0.78$ ( $s, \mathrm{H}-$ $24)$ and $0.77(s, \mathrm{H}-26),{ }^{13} \mathrm{C}-\mathrm{NMR}\left(125 \mathrm{MHz}, \mathrm{CDCl}_{3}\right)$ d (ppm): 177.8 (C-28), 143.7 (C-13), 122.9 (C-12), 79.2 (C-3), 55.4 (C-5), 47.8 (C-9), 46.6 (C-17), 46.1 (C-19), 41.9 (C-14), 41.3 (C-18), 39.5 (C-8), 38.9 (C1), 38.6 (C-4), 37.3 (C-10), 34.0 (C-21), 33.2 (C-29), 32.9 (C-22), 32.6 (C-7), 30.8 (C-20), 28.3 (C-23), 27.9 (C-15), 27.4 (C-2), 26.1 (C-27), 23.7 (C-30), 23.6 (C16), 23.2 (C-11), 18.5 (C-6), 17.2 (C-26), 15.7 (C-24) and $15.5(\mathrm{C}-25)$.

Corosolic acid (5): white powder, ESI-MS $m / z: 471.43$ $[\mathrm{M}-\mathrm{H}]^{-}$corresponding for $\mathrm{C}_{30} \mathrm{H}_{48} \mathrm{O}_{4} \cdot{ }^{1} \mathbf{H}-\mathrm{NMR}$ $\left(500 \mathrm{MHz}, \mathrm{CDCl}_{3}\right) \mathrm{d}(\mathrm{ppm}): 5.15(d, 14.5 \mathrm{~Hz}, \mathrm{H}-$ 12), 3.41 ( $m, \mathrm{H}-2), 2.74(d, 9.5 \mathrm{~Hz}, \mathrm{H}-3), 2.11(d, 11.5$ $\mathrm{Hz}, \mathrm{H}-18), 1.04$ (s, H-27), 0.92 (s, H-23, H25), 0.91 $(d, 7.0 \mathrm{~Hz}, \mathrm{H}-30), 0.82$ (d, $6.0 \mathrm{~Hz}, \mathrm{H}-29), 0.75$ ( $s, \mathrm{H}-$ 26), $0.71(s, \mathrm{H}-24),{ }^{13} \mathrm{C}-\mathrm{NMR}\left(125 \mathrm{MHz}, \mathrm{CDCl}_{3}\right) \mathrm{d}$ (ppm): 178.4 (C-28), 138.3 (C-13), 124.5 (C-12), 82.3 (C-3), 67.2 (C-2), 54.8 (C-5), 52.4 (C-18), 47.1 (C-17), 47.0 (C-9), 46.8 (C-1), 41.7 (C-14), 38.9 (C-8), 38.5 (C-4, C-19 \& C-20), 37.6 (C-10), 36.3 (C-22), 32.6 (C7), 30.2 (C-21), 28.8 (C-23), 27.5 (C-15), 25.6 (C-16), 23.3 (C-27), 22.9 (C-11), 21.1 (C-30), 18.0 (C-6), 17.2 (C-29), 17.0 (C-26), 16.9 (C-25) and 16.4 (C-24).

$\beta$-Sitosterol (6): white powder, $\mathrm{C}_{29} \mathrm{H}_{50} \mathrm{O} .{ }^{1} \mathrm{H}-\mathrm{NMR}$ $\left(500 \mathrm{MHz}, \mathrm{CDCl}_{3}\right) \mathrm{d}(\mathrm{ppm}): 5.35(d, 5.0 \mathrm{~Hz}, \mathrm{H}-6)$, 3.52 ( $m, \mathrm{H}-3), 1.01$ ( $s, \mathrm{H}-18), 0.92(d, 6.5 \mathrm{~Hz}, \mathrm{H}-21)$, 0.85 (d, $7.5 \mathrm{~Hz}, \mathrm{H}-29), 0.83$ (d, $6.5 \mathrm{~Hz}, \mathrm{H}-27), 0.81$ (d, 7.0, Hz, H-26), 0.68 ( $s, \mathrm{H}-19),{ }^{13} \mathrm{C}-\mathrm{NMR}(125 \mathrm{MHz}$, $\mathrm{CDCl}_{3}$ ) d (ppm): 140.9 (C-5), 121.9 (C-6), 72.0 (C3), 56.9 (C-14), 56.2 (C-17), 50.3 (C-9), 46.0 (C-24), 42.5 (C-4 \& C-13), 39.9 (C-12), 37.4 (C-1), 36.7 (C10), 36.3 (C-20), 34.1 (C-22), 32.1 (C-7 \& C-8), 31.8 


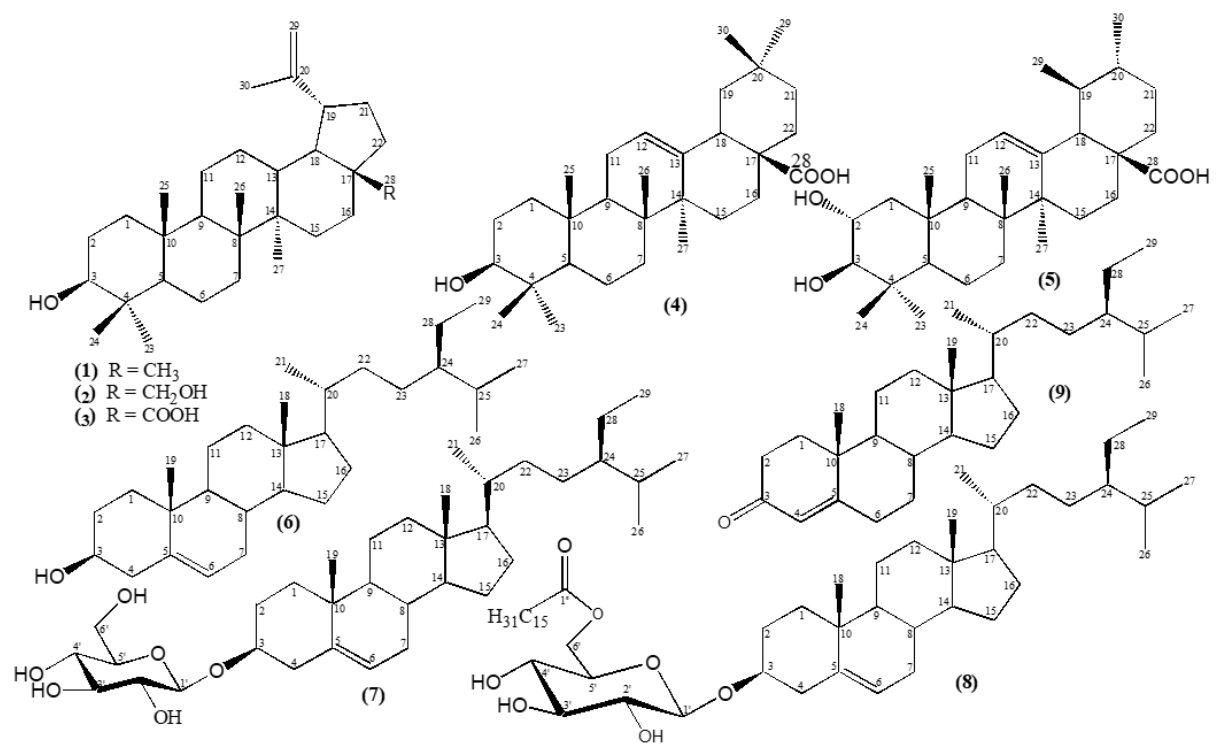

Figure 2: The chemical structures of isolatedcompounds from Lumnitzera littorea leaves.

(C-2), 29.3 (C-25), 28.4 (C-16), 26.3 (C-15), 24.5 (C23), 23.2 (C-28), 21.2 (C-11), 20.0 (C-27), 19.5 (C-26), 19.2 (C-21), 18.9 (C-19), 12.1 (C-29) and 12.0 (C-18). $\beta$-Sitosterol 3-O- $\beta$-D-glucopyranoside (7): white powder, $\mathrm{C}_{35} \mathrm{H}_{60} \mathrm{O}_{6}$. ${ }^{1} \mathbf{H}-\mathrm{NMR}(500 \mathrm{MHz}, \mathrm{DMSO}-$ $\left.d_{6}\right) \mathrm{d}(\mathrm{ppm}): 5.32,(b r s, \mathrm{H}-6), 4.21\left(d, 8.0 \mathrm{~Hz}, \mathrm{H}-1^{\prime}\right)$, 3.12 ( $m, \mathrm{H}-3), 2.89-3.15$ ( $m, \mathrm{H}-2^{\prime}-6$ '), 0.95 ( $\left.s, \mathrm{H}-19\right)$, $0.89(d, 6.5, \mathrm{H}-21), 0.82(d, 7.0 \mathrm{~Hz}, \mathrm{H}-29), 0.81$ (d, 7.0 $\mathrm{Hz}, \mathrm{H}-26), 0.79$ (d, $7.5 \mathrm{~Hz}, \mathrm{H}-27), 0.64(s, \mathrm{H}-18),{ }^{13} \mathrm{C}-$ NMR (125 MHz, DMSO- $\left.d_{6}\right) \mathrm{d}(\mathrm{ppm}): 140.5(\mathrm{C}-5)$, 121.3 (C-6), 100.9 (C-1'), 77.1 (C-3 \& C-3'), 76.8 (C5’), 73.5 (C-2'), 70.2 (C-4'), 61.2 (C-6'), 56.3 (C-14), 55.5 (C-17), 49.7 (C-9), 45.2 (C-24), 41.9 (C-13), 39.0 (C-4), 38.4 (C-12), 36.9 (C-1), 36.3 (C-10), 35.6 (C20), 33.4 (C-22), 31.5 (C-7 \& C-8), 29.3 (C-2), 28.8 (C-25), 27.9 (C-16), 25.5 (C-23), 24.0 (C-15), 22.7 (C28), 20.9 (C-11), 19.8 (C-26), 19.2 (C-19), 19.0 (C-27), 18.7 (C-21), 11.9 (C-29) and 11.8 (C-18).

Stigmast-5-ene-3 $\beta$ - $O$-(6-O-hexadecanoyl- $\beta$-D-

glucopyranoside) (8):white powder, $\mathrm{C}_{51} \mathrm{H}_{90} \mathrm{O}_{7}$. ${ }^{1}$ H-NMR ( $500 \mathrm{MHz}, \mathrm{CDCl}_{3}$ ) d (ppm): 5.37 (d, 5.0 Hz, H-6), 4.50 ( $d d, 12.0,4.5$ Hz, H-6'a), 4.38 (d, 8.0 $\left.\mathrm{Hz}, \mathrm{H}-1^{\prime}\right), 4.26$ (dd, 12.0, $\left.2.0 \mathrm{~Hz}, \mathrm{H}-6^{\prime} \mathrm{b}\right), 3.56$ ( $m$, H-3), 3.34-3.59 (m, H-2'-5'), 2.35 ( $t, 7.5 \mathrm{~Hz}, \mathrm{H}-2^{2}$ ), 1.01 (s, H-19), $0.92(d, 6.5 \mathrm{~Hz}, \mathrm{H}-21), 0.89(d, 7.0 \mathrm{~Hz}$, $\mathrm{H}-29), 0.84(d, 1.5 \mathrm{~Hz}, \mathrm{H}-26), 0.82(d, 4.0 \mathrm{~Hz}, \mathrm{H}-27)$ and $0.68(s, \mathrm{H}-18),{ }^{13} \mathrm{C}-\mathrm{NMR}\left(125 \mathrm{MHz}, \mathrm{CDCl}_{3}\right)$ d (ppm): $174.9\left(\mathrm{C}-1^{2}\right), 140.5$ (C-5), 122.3 (C-6), 101.4 (C-1'), 79.7 (C-3), 76.1 (C-3'), 74.2 (C-5'), 73.8 (C-2'), 70.3 (C-4'), 63.3 (C-6'), 56.9 (C-14), 56.3
(C-17), 50.4 (C-9), 46.0 (C-24), 42.5 (C-13), 39.9 (C-4), 39.1 (C-12), 37.4 (C-11), 36.9 (C-10), 36.3 (C-20), $34.4\left(\mathrm{C}-2^{2}\right), 34.1$ (C-22), 32.1 (C-7, C-8 \& C- $\left.3^{2}\right), 29.7$ (C-1), 29.3 (C-25), 29.3-29.7 (C-4 $\left.{ }^{2}-13^{2}\right)$, 28.4 (C-16), 26.3 (C-23), 25.1 (C-14 $\left.{ }^{2}\right), 24.5$ (C-15), 23.3 (C-28), $22.8\left(\mathrm{C}-15^{2}\right), 21.3$ (C-2), 20.0 (C-29), 19.5 (C-19), 19.2 (C-27), 19.0 (C-21), $14.3\left(\mathrm{C}-16^{2}\right)$, 12.1 (C-26) and 12.0 (C-18).

Stigmast-4-ene-3-one (9): white powder, ESI$\mathbf{M S} m / z: 413.26[\mathrm{M}+\mathrm{H}]^{+}$for $\mathrm{C}_{29} \mathrm{H}_{48} \mathrm{O} .{ }^{\mathbf{1}} \mathbf{H}-\mathrm{NMR}$ $\left(500 \mathrm{MHz}, \mathrm{CDCl}_{3}\right) \mathrm{d}(\mathrm{ppm}): 5.72(s, \mathrm{H}-4), 1.18(s$, H-19), 0.91 (d, $6.5 \mathrm{~Hz}, \mathrm{H}-21), 0.84(t, 7.5 \mathrm{~Hz}, \mathrm{H}-29)$, $0.83(d, 7.0 \mathrm{~Hz}, \mathrm{H}-26), 0.81(d, 7.0 \mathrm{~Hz}, \mathrm{H}-27)$ and 0.71 (s, H-18), ${ }^{13}$ C-NMR (125 MHz, $\left.\mathrm{CDCl}_{3}\right) \mathrm{d}$ (ppm): 199.8 (C-3), 171.9 (C-5), 123.9 (C-4), 56.2 (C-17), 56.0 (C-14), 54.0 (C-9), 46.0 (C-24), 42.5 (C-13), 39.8 (C-12), 38.8 (C-10), 36.3 (C-20), 35.8 (C-1 \& C-8), 34.1 (C-22), 34.0 (C-2), 33.1 (C-6), 32.2 (C-7), 29.3 (C-25), 28.3 (C-16), 26.2 (C-23), 24.3 (C-15), 23.2 (C-28), 21.2 (C-11), 20.0 (C-26), 18.9 (C-21 \& C-27), 17.5 (C-19) and 12.1 (C-18 \& C-29).

\section{In vitro $\alpha$-glucosidase inhibitory assay}

The $\alpha$-glucosidase inhibitory effects of the isolated compounds (1-9) were evaluated. The inhibition \% and $\mathrm{IC}_{50}$ values of all compounds are shown in Table 1 .

The resulting $\mathrm{IC}_{50}$ values indicated that all the compounds, except 8 , showed stronger $\alpha$-glucosidase inhibitory activity than acarbose ( IC $_{50} \quad 127.64 \pm$ 


\begin{tabular}{|c|c|c|c|c|c|c|c|c|c|c|c|c|}
\hline Compound & $\begin{array}{l}\text { Concentration } \\
\mathrm{n}(\mu \mathrm{g} / \mathrm{mL})\end{array}$ & 5 & & 25 & & 50 & & 75 & & 100 & & $\begin{array}{l}\mathrm{IC}_{50} \\
(\mu \mathrm{g} / \mathrm{mL})\end{array}$ \\
\hline 1 & Inhibition (\%) & $\begin{array}{l}30.22 \\
0.48\end{array}$ & \pm & $\begin{array}{l}36.27 \\
0.43\end{array}$ & \pm & $\begin{array}{l}39.38 \\
0.340\end{array}$ & \pm & $\begin{array}{l}41.45 \\
0.75\end{array}$ & \pm & $\begin{array}{l}53.02 \\
0.62\end{array}$ & \pm & $97.95 \pm 0.58$ \\
\hline 2 & & $\begin{array}{l}17.81 \\
0.54\end{array}$ & \pm & $\begin{array}{l}46.79 \\
0.68\end{array}$ & \pm & $\begin{array}{l}60.25 \\
0.72\end{array}$ & \pm & $\begin{array}{l}70.81 \\
0.35\end{array}$ & \pm & $>100$ & & $38.74 \pm 0.63$ \\
\hline 3 & & $\begin{array}{l}24.02 \\
0.28\end{array}$ & \pm & $\begin{array}{l}53.00 \\
0.43\end{array}$ & \pm & $\begin{array}{l}72.67 \\
0.27\end{array}$ & \pm & $\begin{array}{l}89.03 \\
0.18\end{array}$ & \pm & $>100$ & & $28.12 \pm 0.37$ \\
\hline 4 & & $\begin{array}{l}38.51 \\
0.43\end{array}$ & \pm & $\begin{array}{l}55.07 \\
0.53\end{array}$ & \pm & $\begin{array}{l}80.95 \\
0.75\end{array}$ & \pm & $\begin{array}{l}94.41 \\
0.61\end{array}$ & \pm & $>100$ & & $18.82 \pm 0.59$ \\
\hline 5 & & $\begin{array}{l}43.16 \\
0.16\end{array}$ & & $\begin{array}{l}50.76 \\
0.37\end{array}$ & \pm & $\begin{array}{l}84.98 \\
0.43\end{array}$ & \pm & $>100$ & & $>100$ & & $17.86 \pm 0.42$ \\
\hline 6 & & $\begin{array}{l}31.30 \\
0.27\end{array}$ & \pm & $\begin{array}{l}38.30 \\
0.63\end{array}$ & \pm & $\begin{array}{l}63.19 \\
0.21\end{array}$ & \pm & $\begin{array}{l}74.81 \\
0.18\end{array}$ & \pm & $>100$ & & $34.45 \pm 0.34$ \\
\hline 9 & & $\begin{array}{l}28.24 \\
0.17\end{array}$ & & $\begin{array}{l}43.20 \\
0.26\end{array}$ & \pm & $\begin{array}{l}62.75 \\
0.34\end{array}$ & \pm & $\begin{array}{l}78.32 \\
0.53\end{array}$ & \pm & $\begin{array}{l}92.98 \\
0.48\end{array}$ & \pm & $38.18 \pm 0.45$ \\
\hline & $\begin{array}{l}\text { Concentration } \\
\mathrm{n}(\mu \mathrm{g} / \mathrm{mL})\end{array}$ & 10 & & 50 & & 100 & & 150 & & 200 & & $\begin{array}{l}\text { IC50 } \\
(\mu \mathrm{g} / \mathrm{mL})\end{array}$ \\
\hline 7 & Inhibition (\%) & $\begin{array}{l}9.92 \\
0.39\end{array}$ & \pm & $\begin{array}{l}32.52 \\
0.26\end{array}$ & \pm & $\begin{array}{l}49.31 \\
0.17\end{array}$ & \pm & $\begin{array}{l}63.19 \\
0.63\end{array}$ & \pm & $\begin{array}{l}74.81 \\
0.72\end{array}$ & \pm & $\begin{array}{l}114.19 \\
0.61\end{array}$ \\
\hline 8 & & $\begin{array}{l}2.56 \\
0.39\end{array}$ & \pm & $\begin{array}{l}15.63 \\
0.76\end{array}$ & \pm & $\begin{array}{l}32.67 \\
0.35\end{array}$ & \pm & $\begin{array}{l}45.81 \\
0.49\end{array}$ & \pm & $\begin{array}{l}53.67 \\
0.39\end{array}$ & \pm & $\begin{array}{l}174.51 \\
0.58\end{array}$ \\
\hline $\begin{array}{l}\text { Acarbose } \\
\text { (Positive } \\
\text { control) }\end{array}$ & & $\begin{array}{l}4.65 \\
0.35\end{array}$ & \pm & $\begin{array}{l}10.47 \\
0.21\end{array}$ & \pm & $\begin{array}{l}39.54 \\
0.67\end{array}$ & \pm & $\begin{array}{l}62.40 \\
0.64\end{array}$ & \pm & $\begin{array}{l}79.07 \\
0.51\end{array}$ & \pm & $\begin{array}{ll}127.64 \quad \pm \\
0.64\end{array}$ \\
\hline
\end{tabular}

Data are presented as mean $\pm S D$ values of triplicate determinations. A one-way analysis of variance (ANOVA) and positive analysis were done using Duncan's multiple test; significance was set at $P<0.05$.

$0.64 \mathrm{mg} / \mathrm{mL})$. Particularly, one ursane-type triterpene (compound 5) and one oleanane-type triterpene (compound 4) showed outstanding $\alpha$-glucosidase inhibition activities, with $\mathrm{IC}_{50}$ values of $17.86 \pm 0.42$ and $18.82 \pm 0.59 \mu \mathrm{g} / \mathrm{mL}$, respectively. Meanwhile, the other compounds displayed $\alpha$-glucosidase inhibition activities with $\mathrm{IC}_{50}$ values ranging from of $34.00-$ $115.00 \mu \mathrm{g} / \mathrm{mL}$.

\section{DISCUSSION}

The ${ }^{1} \mathrm{H}$ NMR spectrum of compounds 1-5 showed the presence of several singlet signals in the high shielded region at $\mathrm{d}$ 0.71-1.69, that was characteristic of methyl protons. The ${ }^{13} \mathrm{C}$ NMR spectrum of compounds 1-5 revealed 30 carbon signals, including seven methyl carbons, nine methylene carbons, seven methine carbons, and seven non-hydrogenated carbons. The result showed characteristic of a pentacyclic triterpenoid. On the other hand, the skeleton of $\mathbf{1}$ was recognized to be lupane triterpenoid by the
NMR spectra, with the typical olefinic proton signals at $\mathrm{d} 4.56$ (s, H-29b) and 4.68 (brs, H-29a) in the ${ }^{1} \mathrm{H}$ NMR spectrum and two olefinic carbons of the exocyclic double bond at $\mathrm{d} 109.5$ (C-29) and 151.1 (C-20) in the ${ }^{13} \mathrm{C}$ NMR spectrum. Moreover, the assignment of the hydroxyl group at C-3 was performed by the presence of one secondary hydroxyl proton signal at d 3.18 (dd, 11.5, $5.0 \mathrm{~Hz}, \mathrm{H}-3)$, correlating with a carbon signal at $\mathrm{d} 79.1$ (C-3). Thus, 1 was determined as lupeol that was consistent with the reported values in the literature ${ }^{4}$.

The NMR spectra of $\mathbf{2}$ were similar to those of $\mathbf{1}$, including the proton and carbon signals for the terpenoid of lupane skeleton. The ${ }^{1} \mathrm{H}$ NMR spectrum of $\mathbf{2}$ differed from that of $\mathbf{1}$ by having a pair of proton signals at $\mathrm{d} 3.33(d, 11.0 \mathrm{~Hz}, \mathrm{H}-28 \mathrm{~b})$ and $3.80(d, 10.5 \mathrm{~Hz}$, $\mathrm{H}-28 \mathrm{a}$ ), instead of a methyl proton signal at $\mathrm{d} 0.79$ ( $s$, $\mathrm{H}-28)$ as in $\mathbf{1}$. In the ${ }^{13} \mathrm{C}$ NMR spectrum of 2, besides an oxygenated methine carbon signal at $\mathrm{d} 79.1$ (C-3), compound $\mathbf{2}$ had another oxygenated methylene car- 
bon signal at $\mathrm{d} 60.7$ (C-28), thus confirming that there was a second hydroxyl group at C-28 in the structure of 2. Comparison of the spectroscopic data of $\mathbf{2}$ with those in the literature suggested 2 was betulin ${ }^{4}$.

Similar to the NMR spectra of 2 , the ${ }^{1} \mathrm{H}$ NMR and

${ }^{13} \mathrm{C}$ NMR spectra of $\mathbf{3}$ also possessed the signals of a lupane skeleton. However, the ${ }^{1} \mathrm{H}$ NMR spectrum of $\mathbf{3}$ differed from that of $\mathbf{2}$ in the absence of a pair of proton signals at $\mathrm{d} 3.30-3.80$ of $\mathrm{H}-28$ position. It corresponded to the presence of a carboxyl carbon signal at $\mathrm{d} 180.5$, instead of an oxygenated methylene carbon signal at $\mathrm{d} 60.7$ (C-28) as in 2. Thus, compound 3 was betulinic acid whose NMR data were in good compatibility with those in the literature ${ }^{5}$.

The ${ }^{1} \mathrm{H}$ NMR spectrum of compound 4 displayed one olefinic proton signal at d $5.29(t, 3.5 \mathrm{~Hz}, \mathrm{H}-12)$, together with a signal at d $2.83(d d, 13.5,4.0 \mathrm{~Hz}, \mathrm{H}-18)$ which indicated the oleanan-12-ene skeleton. One methine proton signal at d $3.22(d d, 11.5,4.0 \mathrm{~Hz}, \mathrm{H}$ 3) showed that $\mathbf{4}$ had one hydroxyl group. The ${ }^{13} \mathrm{C}$ NMR spectral data exhibited signals at d 122.9 and 143.7, corresponding to the carbons C-12 and C-13, respectively. The signal at $\mathrm{d} 177.8$ was assigned to the carboxyl group at C-28. This data allowed the identification of compound $\mathbf{4}$ as oleanolic acid which is isolated for the first time from Lumnitzera littorea.

The ${ }^{1} \mathrm{H}$ NMR spectrum of compound 5 showed the presence of two doublet methyl signals at d $0.82(d$, $6.0 \mathrm{~Hz}, \mathrm{H}-29)$ and $0.91(d, 7.0 \mathrm{~Hz}, \mathrm{H}-30)$, which were characteristics for ursane skeleton. Furthermore, the olefinic proton signal was observed at $\mathrm{d} 5.15(t d, 14.5$, $3.6 \mathrm{~Hz}, \mathrm{H}-12$ ) along with one methine proton signal at $\mathrm{d} 2.11(d, 11.5 \mathrm{~Hz}, \mathrm{H}-18)$. Two oxygenated methine proton signals at $\mathrm{d} 2.74(d, 9.5 \mathrm{~Hz})$ and $3.41(\mathrm{~m}$, overlapped with the solvent signal) were assigned to $\mathrm{H}$ 3 and $\mathrm{H}-2$, respectively. In the ${ }^{13} \mathrm{C} N M R$ spectrum of 5 showed two oxygenated carbons at d 67.2 (C-2) and $82.3(\mathrm{C}-3)$, two disubstituted double carbons at $\mathrm{d}$ 124.5 (C-12) and 138.3 (C-13), and one carbonyl carbon $\mathrm{d} 178.4$ (C-28). The spectral data were similar to the ones reported for corosolic acid ${ }^{6}$.

The ${ }^{1} \mathrm{H}$ NMR spectrum of compound 6 revealed the presence of six methyl proton signals, including two methyl singlets at d $0.68(s, \mathrm{H}-19)$ and $1.01(s, \mathrm{H}-18)$, four methyl doublets at $\mathrm{d} 0.81(d, 7.0 \mathrm{~Hz}, \mathrm{H}-26), 0.83$ (d, $6.5 \mathrm{~Hz}, \mathrm{H}-27), 0.85$ (d, $7.5 \mathrm{~Hz}, \mathrm{H}-29)$, and 0.92 (d, $6.5 \mathrm{~Hz}, \mathrm{H}-21)$. The olefinic proton signal d $5.35(d, 5.0$ $\mathrm{Hz}, \mathrm{H}-6)$ appeared to be characteristic of the sterols. Furthermore, the proton signal connected to the C-3 hydroxyl group appeared as a multiplet at d $3.52(\mathrm{~m}$, $\mathrm{H}-3)$. The ${ }^{13} \mathrm{C}$ NMR spectrum exhibited 29 carbon signals, including two carbon signals at d 121.9 (C-6) and 140.9 (C-5), characteristic of a double bond and an oxymethine carbon signal at d 72.0 (C-3). Thus, the structure of $\mathbf{6}$ was assigned as $\beta$-sitosterol and was consistent with values reported in the literature ${ }^{7}$. Detailed analysis of NMR spectra of 7 indicated that it also possessed the proton and carbon signals of a $\beta$ sitosterol skeleton. Additionally, the ${ }^{1} \mathrm{H}$ NMR spectrum of 7 confirmed the presence of one $\beta$-glucose unit through a doublet signal at d $4.21(d, 8.0 \mathrm{~Hz}, \mathrm{H}$ 1 '), assigned for anomeric proton, and multiplet signals from $\mathrm{d} 2.89$ to 3.15 , assigned for the carbinol protons of the sugar part. In the ${ }^{13} \mathrm{C} N M R$ spectrum which displayed an anomeric carbon signal at $\mathrm{d} 100.9$ (C-1'), an oxygenated methylene carbon signal at $\mathrm{d}$ 61.2 (C-6') and four oxymethine carbon signals at $\mathrm{d}$ 70.2-77.1 (C-2'-5') of sugar unit were observed. These data confirm that compound 7 was $\beta$-itosterol 3-O$\beta$-D-glucopyranoside ${ }^{8}$.

Similar to the NMR spectra of 7 , the ${ }^{1} \mathrm{H}$ NMR and ${ }^{13} \mathrm{C}$ NMR spectra of $\mathbf{8}$ indicated that it possessed a similar structure to that of $\beta$-sitosterol glucoside. The difference was the presence of signals for a palmitoyl moiety in 8 . The ${ }^{1} \mathrm{H}$ NMR spectrum observed one methylene proton signal adjacent to a carboxyl group at $\mathrm{d} 2.35\left(t, 7.5 \mathrm{~Hz}, \mathrm{H}-2^{2}\right)$, other methylene proton signals at $\mathrm{d}$ 1.20-1.50 (characteristic for a long aliphatic chain), and one terminal methyl proton signal at $\mathrm{d} 0.89\left(d, 7.0 \mathrm{~Hz}, \mathrm{H}-16^{2}\right)$. Moreover, the ${ }^{13} \mathrm{C}$ NMR spectrum revealed 51 carbon signals, including 29 carbons of a $\beta$-itosterol skeleton and 6 carbons of a glucose unit. The assignment of 1 carbon signal at $\mathrm{d}$ $174.9\left(\mathrm{C}-1^{2}\right)$ was determined by the presence of a carboxyl group, as well as carbon signals at d 29.3-29.7 of the methylene carbons and at d 14.3 of a terminal methyl group. Besides that, the HMBC correlations of methylene protons at $\mathrm{d} 4.26(d d, 12.0,2.0 \mathrm{~Hz}, \mathrm{H}-6 \mathrm{cb})$ and $4.50\left(d d, 12.0,4.5 \mathrm{~Hz}, \mathrm{H}-6{ }^{\prime} \mathrm{a}\right)$ with the carbonyl carbon at $\mathrm{d} 174.9\left(\mathrm{C}-1^{2}\right)$ indicated an attachment of the palmitoyl moiety at C-6' of the glucose unit. Comparison of spectroscopic data of $\mathbf{8}$ with those in the literature suggested that 8 was stigmast-5-ene-3 $\beta$-O(6-O-hexadecanoyl- $\beta$-D-glucopyranoside $)^{4}$.

The ${ }^{1} \mathrm{H}$ NMR spectrum of 9 closely resembled that of 6 . In addition, the ${ }^{1} \mathrm{H}$ NMR spectrum confirmed the presence of one olefinic proton signal at d $5.72(s$, $\mathrm{H}-4)$ and the absence of a multiplet proton signal at d 3.10-3.60 of $\mathrm{H}-3$ position. The ${ }^{13} \mathrm{C}$ NMR spectrum showed the carbonyl carbon signal at d 199.8 (C-3) and two olefinic carbon signals at d 171.9 (C-5) and 123.9 (C-4). Based on the spectral data obtained and comparison with literature data, the structure of 9 was confirmed as stigmast-4-ene-3-one ${ }^{9}$.

Although 1-5, 8, and 9 are known compounds, this is the first time their presence in leaves of Lumnitzera 
littorea has been reported. $\alpha$-Glucosidases are a series of enzymes located on the human intestine. The most important carbohydrates in food are hydrolyzed to monosaccharide by $\alpha$-glucosidase, then absorbed into the blood to increase blood glucose level. This is the reason for development of diabetes. The $\alpha$ Glucosidase inhibitors may have the potential to delay or prevent the rise of blood glucose level. However, the mechanism of the inhibitions against $\alpha$ glucosidase has not yet clear.

In our experiments, five compounds of triterpenoids and four compounds of steroids from Lumnitzera littorea showed different activity against $\alpha$-glucosidase (Table 1). From the structures of compounds 1-3, we can infer that the $\alpha$-glucosidase inhibitory acitvity is strengthened when the methylene group at C-28 is altered to an oxygenated methylene or a carboxylic group. As the result, the $\mathrm{IC}_{50}$ values of lupeol (1), betulin (2) and betulinic acid (3) were $97.95 \pm 0.85$, $38.74 \pm 0.63$ and $28.82 \pm 0.37 \mu \mathrm{g} / \mathrm{mL}$, respectively. Furthermore, a carboxylic acid group or a $\mathrm{CH}_{2}-\mathrm{OH}$ group at $\mathrm{C}-17$ is important for the action of compounds 2-5.

Comparison of the chemical structures and the $\alpha$ glucosidase inhibitory activity indicates that the presence of a hydroxyl group at $\mathrm{C}-3$ plays an important role in the $\alpha$-glucosidase inhibitory activity. Thus, the data from this study also demonstrated that the $\mathrm{IC}_{50}$ values of compounds 2-6 were lower than those of compounds 7 and 8-9.Of note, it is interesting that for $\mathbf{9}$ it is not an $-\mathrm{OH}$ group but an $=\mathrm{O}$ group. However, an oxygen is not enough at 7 and 8 have low activity. This could be ascribed to the more bulky structure of the inhibitor. Thus, the presence of one $\beta$-glucose unit at $\mathrm{C}-3$ of $\beta$ sitosterol 3-O- $\beta$-D-glucopyranoside (7) or the attachment of the palmitoyl moiety at C- 6 ' of the glucose unit of stigmast-5-ene-3 $\beta$-O-(6-O-hexadecanoyl- $\beta$ D-glucopyranoside) (8) decreased the $\alpha$-glucosidase inhibitory activity. This demonstrated that the $\mathrm{IC}_{50}$ values of compounds 6-8 had increased to $34.45 \pm$ $0.34,114.19 \pm 0.61$ and $174.51 \pm 0.58 \mu \mathrm{g} / \mathrm{mL}$, respectively.

When the methylene group at C-2 was altered to a hydroxyl group, the $\alpha$-glucosidase inhibitory activity increased. This also indicated that $\mathrm{IC}_{50}$ values of corosolic acid (5), as the most effective compound, displayed a significantly inhibitory activity against $\alpha$ glucosidase with $\mathrm{IC}_{50}$ values of $17.86 \pm 0.42 \mu \mathrm{g} / \mathrm{mL}$.

\section{CONCLUSIONS}

In the investigation of the chemical constituents of Lumnitzera littorea leaves, nine compounds were iso- lated. There were five triterpenoids: lupeol (1), betulin (2), betulinic acid (3), oleanolic acid (4), and corosolic acid (5). As well, there were four steroids: $\beta$ sitosterol (6), $\beta$-sitosterol 3-O- $\beta$-D-glucopyranoside (7), stigmast-5-ene-3 $\beta$-O-(6-O-hexadecanoyl- $\beta$-Dglucopyranoside) (8), and stigmast-4-ene-3-one (9). Although these compounds were already known in other species, this is the first time they were reported in Lumnitzera littorea. All of them were evaluated for $\alpha$-glucosidase inhibitory activity and among them, corosolic acid was the most potent inhibitor with $\mathrm{IC}_{50}$ values of $17.86 \pm 0.42 \mu \mathrm{g} / \mathrm{mL}$, closely followed by oleanolic acid. Based on our report, one may expect compound 4 (with a hydroxyl group added at C-3) to be very active.

\section{ABBREVIATIONS}

${ }^{13}$ C NMR: Carbon-13 nuclear magnetic resonance

${ }^{1}$ H NMR: Proton nuclear magnetic resonance

CC: column chromatography

$\mathrm{CDCl}_{3}$ : chloroform- $d$.

DMSO: Dimethyl sulfoxide $\left(\mathrm{CD}_{3} \mathrm{SOCD}_{3}\right)$

HMBC: Heteronuclear multiple bond correlation

TLC: Thin layer chromatography

\section{COMPETING INTERESTS}

The authors declare that they have no conflicts of interest.

\section{AUTHORS' CONTRIBUTIONS}

Nguyen Thi Le Thuy and Pham Thi Thuy have contributed in conducting experiments, getting hold of data and writting the manuscript. Poul Erik Hansen and Nguyen Kim Phi Phung have contributed significantly explanation of data and revissing the manuscript.

\section{ACKNOWLEDGMENTS}

This research is funded by HoChiMinh City Open University.

\section{REFERENCES}

1. Saad S, Taher M, Susanti D, Qaralleh H, Rahim NA, Abdul R. Antimicrobial activity of mangrove plant (Lumnitzera littorea). Asian Pacific Journal of Tropical Medicine. 2011;4:523-5. Available from: Doi:10.1016/s1995-7645(11)60138-7.

2. Thuy NTL, Hung QT, Duc NT, Thuy PT, Hung LV, Hansen PE, et al. Flavonoids from the leaves of Lumnitzera littorea. Vietnam Journal of Chemistry. 2017;55:606-610.

3. Apostolidis E, Kwon Y, Shetty K. Inhibitory potential of herb, fruit and fungal-enriched cheese against key enzymes linked to type 2 diabetes and hypertension. Innovative Food Science \& Emerging Technologies. 2007;8:46-54. Available from: DOI: $10.1016 /$ j.ifset.2006.06.001. 
4. Tian M, Dai H, Li X, Wang B. Chemical constituents of marine medicinal mangrove plant Sonneratia caseolaris. Chinese Journal of Oceanology and Limnology. 2009;27:288-96. Available from: DOI:10.1007/s00343-009-9138-7.

5. Adrielli T, Ariela MB, Catarina CB, Alexandre B, Caio MM, Valdir C. Chemical composition, antibacterial and antimycoplasma activities of four Eugenia species growing in Brazil. The Journal of Medical Research. 2017;11:596-602.

6. Ahmed ME, Azza AK, Soad AB, Hesham MS. Chemical constituents of ornamental pomegranate and its antioxidant and anti-inflammatory activites in comparison with edible pomegranate. Journal of Pharmacognosy and Phytochemistry.
2016;5:88-94.

7. Venkata SP, Indra P. Isolation of stigmasterol and ?-sitosterol from the dichloromethane extract of Rubus suavissimus. International Current Pharmaceutical Journal. 2012;1:239-42.

8. Tania P, Kar HK. Isolation and Characterization of ?-sitosterol-3O-?-D-glucoside from the extract of the flowers of Viola odorata. British Journal of Pharmaceutical Research. 2017;16:1-8. Available from: Doi:10.9734/bjpr/2017/33160.

9. Kyun L, Min AK, Seung YL, Jong KH, Jei HL, Kang RL. Phytochemical constituents of Schizonepeta tenuifolia Briquet. Natural Product Sciences. 2008;14:100-6. 